\title{
Prediction of Ligand Binding Affinity Using a Multiple-Conformations- Multiple-Protonation Scheme: Application to Estrogen Receptor $\alpha$
}

\author{
Miho Y. Mizutani,* Yoshihiro Takamatsu, Tazuko Ichinose, and Akiko Itai \\ Institute of Medicinal Molecular Design, Inc.; 5-24-5 Hongo, Bunkyo-ku, Tokyo 113-0033, Japan.
}

Received September 8, 2011; accepted November 24, 2011; published online December 2, 2011

\begin{abstract}
A fast method that can predict the binding affinities of chemicals to a target protein with a high degree of accuracy will be very useful in drug design and regulatory science. We have been developing a scoring function for affinity prediction, which can be applied to extensive protein systems, and also trying to generate a prediction scheme that specializes in each target protein, with as high a predictive power as possible. In this study, we have constructed a prediction scheme with target-specific scores for estimating ligand-binding affinities to human estrogen receptor $\alpha(\mathrm{ER} \alpha)$, considering the major conformational change between agonist- and antagonist-bound forms and the change in protonation states of histidine at the ligand-binding site. The generated scheme calibrated with fewer training compounds ( 23 for the agonist-bound form, 17 for the antagonist-bound form) demonstrated good predictive power (a predictive $r^{2}$ of 0.83 for 154 validation compounds); this was also true for compounds with frameworks that were quite different from those of the training compounds. Our prediction scheme will be useful in drug development targeting ER $\alpha$ and in primary screening of endocrine disruptors, and provides a successful method of affinity prediction considering the major conformational changes in a protein.
\end{abstract}

Key words binding affinity; prediction score; docking; protein conformational change; protonatioin state; estrogen receptor

In current drug discovery research, methods of searching for promising seed structures efficiently and optimizing them rationally decide the outcome of pharmaceutical development. The limitations of the high-throughput screening (HTS) technique are becoming clear, and accordingly, rational approaches to seed development by means of computational chemistry have been receiving attention again. In particular, in silico drug design based on the three-dimensional (3D) structure of a target protein has been enthusiastically attempted. ${ }^{1-3)}$ The docking technique, which is the core technique of structurebased drug design (SBDD), has improved considerably over nearly three decades, ${ }^{4,5)}$ although difficult problems such as protein flexibility and consideration of water molecules remain. Meanwhile, another important technique, a prediction score for the binding affinities of small compounds to a target protein, ${ }^{6}$ has not yet met the requirements for accuracy and computational cost. ${ }^{4,7)}$

One approach for predicting binding affinity is to try to estimate the binding free energy rigorously, e.g., the free energy perturbation (FEP) technique, ${ }^{8)}$ linear interaction energy method, ${ }^{9)}$ and Poisson-Boltzmann equation methods. ${ }^{10,11)}$ However, these rigorous methods have a large computational cost for every ligand-protein complex and would not be suitable for sequential evaluation of a number of compounds. Moreover, the FEP method can be adopted only to compare energies between ligands with the same structural frames. A number of empirical scores have also been developed. ${ }^{4,12}$ These scores can handle many compounds because of their lower computational cost and are able to evaluate true actives as higher-ranking groups on the prediction list in many cases reported so far. However, the predictive powers of these empirical methods vary depending on the target proteins; furthermore, they are still insufficient in terms of accurate prediction of binding free energy and correct ranking of compounds by possible activities. In addition to the above methods, approaches based on quantitative structure-activity relationships (QSAR), which do not use the interaction energy between a target protein and compounds, have been developed, e.g., CoMFA, ${ }^{13)}$ Raptor, ${ }^{14)}$ and 6D-QSAR. ${ }^{15)}$ Although these methods display high predictive powers in every protein system, their resulting predictive model depends largely on the training compounds and alignment of ligands in the (putative) protein cavity, which are modeled by hand using the cocrystal structures of analogous ligands or predicted by docking simulation. If either the binding affinity data for a sufficient number of known ligands or the reliable alignment of training and test compounds are unavailable, the predictive power will be considerably reduced, or the prediction model itself cannot be constructed.

We have been developing an empirical scoring function for estimating the binding free energy in universal protein systems. $^{16,17)}$ One of the characteristics of our scoring function, KeyScore, ${ }^{17)}$ is a way to evaluate the desolvation energy; it displayed sufficient predictive power in a variety of test protein systems. However, satisfactory performance was not shown in some cases, perhaps because changes in the internal energy of the ligand and hydrogen bond (H-bond) formation among protein, ligand, and water molecules were not considered, and problems of protein flexibility, entropic effects, and treatment of water molecules were not solved. Nevertheless, it should be very difficult at the current level of physical chemistry to solve all these problems appropriately with low computational cost, and to demonstrate high predictive power on the basis of only one prediction equation in extensive protein systems. Thus, in addition to the universal score, we have decided to attempt to generate a prediction scheme that specializes in each target protein or protein family, with as high a predictive power as possible (a so-called targeted score ${ }^{18,19}$ ). If fewer training compounds are required to generate the prediction scheme, and the dependency on the structural frameworks of the training set can be reduced, such an approach will be as useful as the universal score. 


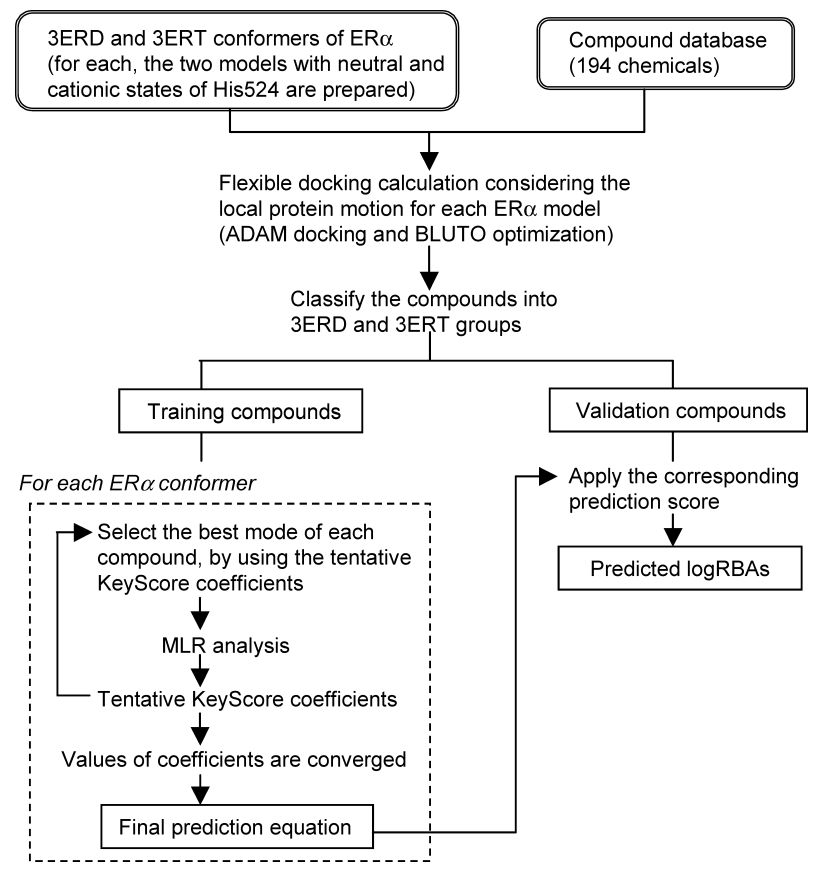

Fig. 1. Flowchart of Our Scheme for Generating and Validating the ER $\alpha$-Targeted Score

In our prediction scheme, the ADAM method ${ }^{20-23)}$ has been used as a docking engine between ligands and the target protein. Since ADAM considers local induced-fit motions, it has the advantage that the docking accuracy for foreign ligands, which are not included in the cocrystal used as the target protein structure, is comparable with that for the cognate ligand in the cocrystal. ${ }^{22}$ ADAM has been applied to dozens of inhouse and collaborative drug-discovery studies, including the virtual screening of aldose reductase, ${ }^{24)}$ acetylcholinesterase, ${ }^{23)}$ the androgen receptor, ${ }^{25)}$ and IKK- $\beta,{ }^{26)}$ and novel ligands with various scaffolds and sufficient activities $\left(\mathrm{IC}_{50}=10^{-6}\right.$ to $\left.10^{-8} \mathrm{M}\right)$ were identified in most cases. In the case of IKK- $\beta$, pharmaceutical development has advanced, and a phase IIa study is now under way.

In this study, human estrogen receptor $\alpha(\mathrm{ER} \alpha)$ was selected as the target of the first trial for generating the targeted score. ER is a ligand-activated transcription factor involved in regulating the cell growth, proliferation, and differentiation of a diverse range of tissues; it is a clinical target for the treatment of hormone-dependent breast cancer, uterine cancer, prostate cancer, and osteoporosis. ${ }^{27)}$ On the other hand, ER is considered to be the main target of endocrine-disrupting chemicals. ${ }^{28)}$ An in silico approach to predicting the binding affinities of various chemicals with a high degree of accuracy has been required and studied in terms of regulatory science as well as pharmaceutical development. ${ }^{14,15,29-36)}$ A number of cocrystal structures of $\mathrm{ER} \alpha$ have been elucidated, and it is known that the ligand-binding cavity of the agonist-bound form of $E R \alpha$ is closed and that of the antagonist-bound form is open to solvents. ${ }^{37,38)}$ Tools that can perform docking calculations while predicting drastic changes in protein conformation, including major movements of backbone atoms, have not yet been reported, ${ }^{39)}$ and ADAM also does not have such functionality. Therefore, we have attempted to perform docking calculation and predict binding affinities using cocrystal structures of both agonist-bound and antagonist-bound forms of $\mathrm{ER} \alpha$. Also, several protonation states for the histidine at the ligand-binding site would be possible depending on the bound ligands, so we intended to take into account this factor in generating the prediction scheme. Furthermore, KeyScore has been improved to estimate explicitly the contribution of changes in ligand conformational energy and H-bonding formation from the isolated to the bound state.

\section{Experimental}

A flowchart of our scheme for generating the ER $\alpha$-targeted score is shown in Fig. 1.

Cocrystal Structures of ER $\alpha$ and Preparation of Compound Database The two cocrystal structures of human ER $\alpha$ were taken from the Protein Data Bank (PDB). One was a complex structure with the pure agonist diethylstilbestrol (DES; PDB code 3ERD), and the other was that with the antagonist 4-hydroxytamoxifen (OHT; PDB code 3ERT). ${ }^{38)}$ In both cocrystal structures, all water molecules were removed except for a structurally conserved one, which forms H-bonds with Glu353 and Arg394. For the side chain of His524, a neutral state (only the $\varepsilon$ position is protonated) and a cationic state (both nitrogens are protonated) were prepared for each protein conformer. As a result, a total of four ER $\alpha$ structures were used in our calculation.

A total of 194 compounds were selected in terms of structural diversity and range of binding affinity, from the binding data set measured by the Chemicals Evaluation and Research Institute (Japan). ${ }^{40)}$ The binding affinities of the chemicals in the data set were determined by receptor binding assay using recombinant human ER $\alpha$. The relative binding affinity (RBA) of each test chemical was calculated using the following equation:

$$
\mathrm{RBA}=\left(\mathrm{IC}_{50} \text { for } \mathrm{E} 2\right) /\left(\mathrm{IC}_{50} \text { for test chemical }\right) \times 100
$$

(E2: $17 \beta$-estradiol)

Compounds that may need rather large induced-fit motion from the 3ERD or 3ERT conformers for binding were excluded (e.g., kepone). The most potent chemicals (e.g., E2, OHT) have $\log$ RBAs of more than 2.0, and the lowest $\log$ RBA is -3.41 ( $p$-isopropylphenol). In addition, 65 non-binders were included. The list of all compounds used in our calculation is shown in Table 1.

The chemical formulae in the compound set were converted to $3 \mathrm{D}$ structures using our program Key3D, ${ }^{41)}$ and MNDOMulliken atomic charges and KMF force field types (our original types) were assigned to all atoms in each compound. All possible stereoisomers were generated, and the possibility of ring-opening for $\alpha$-naphtholphthalein was also taken into account. The 14-membered aliphatic rings in zearalenol and its analogs are very flexible, and several stable ring conformations should be considered for successful docking and scoring; therefore, we prepared 30 conformers for each compound using Key3D. The other attributes necessary for ADAM docking, i.e., H-bonding atom types and settings of bond rotation, were assigned by our EVE-MAKE program. ${ }^{23)}$

Docking to Multiple-Conformations-Multiple-Protonation (MCMP) Target For each of the four ER $\alpha$ models, a $3 \mathrm{D}$ grid with a regular interval of $0.4 \AA$ and a vdW-offset of $0.3 \AA$ (i.e., the vdW energy curve for each atom pair is shifted by $0.3 \AA$ to enlarge the cavity ${ }^{23)}$ was generated inside 
Table 1. Experimental and Calculated $\log$ RBAs for Training and Validation Compounds

\begin{tabular}{|c|c|c|c|c|c|}
\hline \multirow{2}{*}{ Name } & \multirow{2}{*}{ Cas No. } & \multirow{2}{*}{ Model $^{a)}$} & \multicolumn{3}{|c|}{$\log \mathrm{RBA}$} \\
\hline & & & Expt. & Calc. & Deviation \\
\hline \multicolumn{6}{|l|}{ Training compounds } \\
\hline 17ß-Estradiol (E2) & $50-28-2$ & 3ERD & 2.10 & 1.75 & 0.35 \\
\hline Estrone & $53-16-7$ & 3ERD & 1.65 & 1.41 & 0.24 \\
\hline Estrone 3-methyl ether & $1624-62-0$ & 3ERD & -0.84 & -0.62 & 0.22 \\
\hline 16-Epiestriol & $547-81-9$ & 3ERD & 0.88 & 0.95 & 0.07 \\
\hline $6 \alpha$-Hydroxyestradiol & $1229-24-9$ & 3ERD & 1.78 & 0.79 & 0.99 \\
\hline $6 \beta$-Hydroxytestosterone & $62-99-7$ & 3ERD & N.B. ${ }^{b)}$ & -2.26 & $1.74^{c)}$ \\
\hline 4-Ethylphenol & $123-07-9$ & 3ERD & N.B. & -3.52 & 0.48 \\
\hline p-tert-Butylphenol & $98-54-4$ & 3ERD & -2.63 & -2.79 & 0.16 \\
\hline 4-tert-Octylphenol & $140-66-9$ & 3ERD & -0.91 & -0.98 & 0.07 \\
\hline 4-(1-Adamantyl)phenol & 29799-07-3 & 3ERD & 0.23 & 0.20 & 0.03 \\
\hline 4-Cyclohexylresorcinol & $2138-20-7$ & 3ERD & -1.83 & -1.20 & 0.63 \\
\hline 4'-Hydroxy-4-biphenylcarboxylic acid & $58574-03-1$ & 3ERD & -2.52 & -3.45 & 0.93 \\
\hline Bisphenol A & $80-05-7$ & 3ERD & -0.71 & -1.21 & 0.50 \\
\hline Hexestrol & $84-16-2$ & 3ERD & 1.57 & 1.27 & 0.30 \\
\hline Diethylstilbestrol (DES) & $56-53-1$ & 3ERD & 1.95 & 2.27 & 0.32 \\
\hline $2,2^{\prime}, 4,4^{\prime}$-Tetrahydroxybenzophenone & $131-55-5$ & 3ERD & -1.03 & -1.94 & 0.91 \\
\hline 4-Fluorobiphenyl & $324-74-3$ & 3ERD & N.B. & -4.20 & 0.20 \\
\hline Daidzein & $486-66-8$ & 3ERD & -0.74 & -0.59 & 0.15 \\
\hline $3^{\prime}, 4^{\prime}, 7-$ Trihydroxyisoflavone & $485-63-2$ & 3ERD & -1.42 & 0.39 & 1.81 \\
\hline 2-Hydroxy-9-fluorenone & $6949-73-1$ & 3ERD & -2.71 & -2.93 & 0.22 \\
\hline Sesamol & $533-31-3$ & 3ERD & N.B. & -3.66 & 0.34 \\
\hline Coumestrol & $479-13-0$ & 3ERD & -0.58 & -1.71 & 1.13 \\
\hline Atrazine & $1912-24-9$ & 3ERD & N.B. & -3.73 & 0.27 \\
\hline 4-sec-Butylphenol & $99-71-8$ & 3ERT & -2.75 & -3.26 & 0.51 \\
\hline 3-tert-Butylphenol & $585-34-2$ & 3ERT & N.B. & -3.20 & 0.80 \\
\hline 2-Ethylphenol & $90-00-6$ & 3ERT & N.B. & -4.83 & 0.83 \\
\hline$p$-Hydroxybenzoic acid & $99-96-7$ & 3ERT & N.B. & -5.04 & 1.04 \\
\hline 4,4'-Cyclohexylidenebisphenol & $843-55-0$ & 3ERT & -0.67 & -0.91 & 0.24 \\
\hline Bibenzyl & $103-29-7$ & 3ERT & N.B. & -3.42 & 0.58 \\
\hline Benzyl 4-hydroxyphenyl ketone & 2491-32-9 & 3ERT & -2.41 & -2.28 & 0.13 \\
\hline 4-Hydroxytamoxifen (OHT) & $68047-06-3$ & 3ERT & 2.38 & 2.38 & 0.00 \\
\hline Chlorotrianisene & $569-57-3$ & 3ERT & 0.24 & -0.11 & 0.35 \\
\hline Triphenylethylene & $58-72-0$ & 3ERT & -1.13 & -1.19 & 0.06 \\
\hline 1,3,5-Tris(4-hyrdroxyphenyl)benzene & $15797-52-1$ & 3ERT & -0.43 & 0.48 & 0.91 \\
\hline Clomiphene citrate (cis and trans mixture) & $50-41-9$ & 3ERT & 1.57 & 1.18 & 0.39 \\
\hline 4,4'-Dimethylbenzil & $3457-48-5$ & 3ERT & N.B. & -3.49 & 0.51 \\
\hline 4-Methoxybenzophenone & $611-94-9$ & 3ERT & N.B. & -3.02 & 0.98 \\
\hline Bromotriphenylethylene & $1607-57-4$ & 3ERT & 0.43 & -0.75 & 1.18 \\
\hline Dicoumarin & $66-76-2$ & 3ERT & N.B. & -3.19 & 0.81 \\
\hline$\alpha$-Naphtholbenzein & $6948-88-5$ & 3ERT & -0.45 & -0.61 & 0.16 \\
\hline \multicolumn{6}{|l|}{ Validation compounds } \\
\hline $17 \alpha$-Estradiol & $57-91-0$ & 3ERD & 1.90 & 1.51 & 0.39 \\
\hline Estriol & $50-27-1$ & 3ERD & 1.52 & 1.17 & 0.35 \\
\hline Ethynyl estradiol & $57-63-6$ & 3ERD & 2.15 & 1.72 & 0.43 \\
\hline 3-Deoxyestrone & $53-45-2$ & 3ERD & -0.94 & -0.81 & 0.13 \\
\hline Testosterone & $58-22-0$ & 3ERD & N.D. ${ }^{d)}$ & -0.92 & $2.58^{e)}$ \\
\hline p-Butylphenol & $1638-22-8$ & 3ERD & -2.84 & -2.42 & 0.42 \\
\hline$p$-Isopropylphenol & $99-89-8$ & 3ERD & -3.41 & -2.91 & 0.50 \\
\hline p-tert-Amylphenol & $80-46-6$ & 3ERD & -1.76 & -1.95 & 0.19 \\
\hline 4-Cyclohexylphenol & $1131-60-8$ & 3ERD & -1.40 & -1.22 & 0.18 \\
\hline 2,4-Di-tert-butylphenol & $96-76-4$ & 3ERD & -2.81 & -2.22 & 0.59 \\
\hline Thymol & $89-83-8$ & 3ERD & N.B. & -4.00 & 0.00 \\
\hline Butylparaben & $94-26-8$ & 3ERD & -1.75 & -1.67 & 0.08 \\
\hline Propylparaben & $94-13-3$ & 3ERD & -2.03 & -2.18 & 0.15 \\
\hline Isopropyl-4-hydroxybenzoate & $4191-73-5$ & 3ERD & -1.34 & -2.07 & 0.73 \\
\hline 4-Hydroxybenzoic acid isoamyl estar & $6521-30-8$ & 3ERD & -1.62 & -1.64 & 0.02 \\
\hline 4-Ethoxyphenol & $622-62-8$ & 3ERD & N.B. & -4.00 & 0.00 \\
\hline Dihydroxycinnamic acid methyl ester & $63177-57-1$ & 3ERD & -2.74 & -1.78 & 0.96 \\
\hline
\end{tabular}


Table 1. Experimental and Calculated $\log$ RBAs for Training and Validation Compounds

\begin{tabular}{|c|c|c|c|c|c|}
\hline \multirow{2}{*}{ Name } & \multirow{2}{*}{ Cas No. } & \multirow{2}{*}{ Model $^{a)}$} & \multicolumn{3}{|c|}{$\log \mathrm{RBA}$} \\
\hline & & & Expt. & Calc. & Deviation \\
\hline Ethyl 2,4-dihydroxy-6-methylbenzoate & $2524-37-0$ & 3ERD & -2.82 & -2.44 & 0.38 \\
\hline 4-Hexanoylresorcinol & $3144-54-5$ & 3ERD & -2.36 & -1.64 & 0.72 \\
\hline Diethylphthalate & $84-66-2$ & 3ERD & N.B. & -4.00 & 0.00 \\
\hline Diisobutyl phthalate & $84-69-5$ & 3ERD & -3.35 & -3.16 & 0.19 \\
\hline Dicyclohexyl phthalate & $84-61-7$ & 3ERD & -2.98 & -2.83 & 0.15 \\
\hline Butylbenzyl phthalate & $85-68-7$ & 3ERD & -2.50 & -2.08 & 0.42 \\
\hline Diethyl terephthalate & $636-09-9$ & 3ERD & N.B. & -4.00 & 0.00 \\
\hline Flutamide & $13311-84-7$ & 3ERD & N.B. & -4.00 & 0.00 \\
\hline Linuron & $330-55-2$ & 3ERD & N.B. & -4.00 & 0.00 \\
\hline Alachlor & $15972-60-8$ & 3ERD & N.B. & -3.37 & 0.63 \\
\hline 3,4,5-Trimethylphenol & $527-54-8$ & 3ERD & N.B. & -4.00 & 0.00 \\
\hline Propoxur & $114-26-1$ & 3ERD & N.B. & -4.00 & 0.00 \\
\hline Mecprop & $93-65-2$ & 3ERD & N.B. & -4.00 & 0.00 \\
\hline 1,1-Bis(4-hydroxyphenyl)-iso-butane & $1844-00-4$ & 3ERD & -0.59 & -0.51 & 0.08 \\
\hline 4-Chloro-1,1'-biphenyl & $2051-62-9$ & 3ERD & -2.98 & -4.00 & 1.02 \\
\hline p-Phenylphenol & $92-69-3$ & 3ERD & -1.76 & -2.13 & 0.37 \\
\hline Phloroglucide & $491-45-2$ & 3ERD & N.B. & -3.25 & 0.75 \\
\hline 4-(4-Bromophenyl)phenol & $29558-77-8$ & 3ERD & -1.02 & -2.20 & 1.18 \\
\hline 4'-Methoxy-biphenyl-4-ol & $16881-71-3$ & 3ERD & -1.44 & -1.47 & 0.03 \\
\hline 4'-Hydroxy-4-biphenylcarbonitrile & $19812-93-2$ & 3ERD & -2.84 & -1.90 & 0.94 \\
\hline $3,3^{\prime}, 5,5^{\prime}$-Tetramethyl-(1,1'-biphenyl)-4,4'-diol & $2417-04-1$ & 3ERD & -2.38 & -1.49 & 0.89 \\
\hline 4,4'-Dihydroxydiphenylmethane & $620-92-8$ & 3ERD & -1.14 & -2.45 & 1.31 \\
\hline 4,4'-Ethylidenebisphenol & $2081-08-5$ & 3ERD & -1.15 & -1.74 & 0.59 \\
\hline 4- $\alpha$-Cumylphenol & $599-64-4$ & 3ERD & -0.97 & -1.17 & 0.20 \\
\hline 2,2-Bis(4-hydroxyphenyl)-4-methyl- $n$-pentane & $6807-17-6$ & 3ERD & 0.45 & -0.02 & 0.47 \\
\hline Tris(hydroxyphenyl)ethane & $27955-94-8$ & 3ERD & -1.39 & -1.52 & 0.13 \\
\hline 2-[Bis(4-hydroxyphenyl)methyl]benzylalcohol & $81-92-5$ & 3ERD & -0.92 & -1.40 & 0.48 \\
\hline Clofoctol & $37693-01-9$ & 3ERD & -2.21 & -2.15 & 0.06 \\
\hline 4,4'-Sulfonyldiphenol & $80-09-1$ & 3ERD & -2.26 & -2.16 & 0.10 \\
\hline 2,2-Bis(4-hydroxy-3-methylphenyl)propane & $79-97-0$ & 3ERD & -0.89 & -0.85 & 0.04 \\
\hline$o, p^{\prime}-\mathrm{DDD}$ & $53-19-0$ & 3ERD & -2.24 & -2.84 & 0.60 \\
\hline 4-Chloro- $\alpha$-phenyl-benzenemethanol & $119-56-2$ & 3ERD & N.B. & -4.00 & 0.00 \\
\hline Dehydrostilbestrol & $84-17-3$ & 3ERD & 1.31 & 1.14 & 0.17 \\
\hline$p$-Anisil & $1226-42-2$ & 3ERD & N.B. & -3.29 & 0.71 \\
\hline trans-Stilbene & $103-30-0$ & 3ERD & N.B. & -3.31 & 0.69 \\
\hline Resveratrol & $501-36-0$ & 3ERD & -2.50 & -0.73 & 1.77 \\
\hline Benzophenone & $119-61-9$ & 3ERD & N.B. & -4.00 & 0.00 \\
\hline 2-Hydroxybenzophenone & $117-99-7$ & 3ERD & N.B. & -3.46 & 0.54 \\
\hline 4,4'-Dihydroxybenzophenone & $611-99-4$ & 3ERD & -1.77 & -2.69 & 0.92 \\
\hline 2,4-Dihydroxybenzophenone & $131-56-6$ & 3ERD & -1.86 & -1.32 & 0.54 \\
\hline $2,4,4^{\prime}$-Trihydroxybenzophenone & $1470-79-7$ & 3ERD & -1.13 & -1.03 & 0.10 \\
\hline $2,3,4,4^{\prime}$-Tetrahydroxybenzophenone & $31127-54-5$ & 3ERD & -1.60 & -1.20 & 0.40 \\
\hline 4-Fluoro-4'-hydroxybenzophenone & $25913-05-7$ & 3ERD & -2.50 & -2.49 & 0.01 \\
\hline 4-Phenoxyphenol & $831-82-3$ & 3ERD & -1.24 & -1.83 & 0.59 \\
\hline 4-Hydroxychalcone & $20426-12-4$ & 3ERD & -2.91 & -1.95 & 0.96 \\
\hline 4-Hydroxyazobenzene & $1689-82-3$ & 3ERD & -1.13 & -0.89 & 0.24 \\
\hline 4,4'-(1,3-Adamantanediyl)diphenol & $37677-93-3$ & 3ERD & -0.42 & -0.63 & 0.21 \\
\hline Isoliquiritigenin & $961-29-5$ & 3ERD & -1.28 & -0.43 & 0.85 \\
\hline Diphenyl-p-phenylenediamine & $74-31-7$ & 3ERD & -1.87 & -1.74 & 0.13 \\
\hline$S$-(1-Methyl-1-phenylethyl)piperidine-1-carbothioate & $61432-55-1$ & 3ERD & N.B. & -2.91 & 1.09 \\
\hline Leptophos & $21609-90-5$ & 3ERD & -2.72 & -2.70 & 0.02 \\
\hline 2-(4-Hydroxyphenyl)-5-pyrimidinol & $142172-97-2$ & 3ERD & N.B. & -3.01 & 0.99 \\
\hline 4-Nitrobenzophenone & $1144-74-7$ & 3ERD & N.B. & -4.00 & 0.00 \\
\hline (1,1'-Biphenyl)-4-carboxaldehyde & $3218-36-8$ & 3ERD & N.B. & -4.00 & 0.00 \\
\hline trans-4-Hydroxystilbene & $6554-98-9$ & 3ERD & -1.88 & -1.06 & 0.82 \\
\hline 4,4'-Biphenyldicarboxylic acid & $787-70-2$ & 3ERD & N.B. & -4.00 & 0.00 \\
\hline trans-4-Stilbenecarboxaldehyde & $40200-69-9$ & 3ERD & N.B. & -3.14 & 0.86 \\
\hline 2-(4-Chlorophenyl)-1,1-diphenylethanol & $109936-21-2$ & 3ERD & -2.55 & -2.56 & 0.01 \\
\hline $4,4^{\prime}$-Difluorobenzil & $579-39-5$ & 3ERD & N.B. & -4.00 & 0.00 \\
\hline
\end{tabular}


Table 1. Experimental and Calculated $\log$ RBAs for Training and Validation Compounds

\begin{tabular}{|c|c|c|c|c|c|}
\hline \multirow{2}{*}{ Name } & \multirow{2}{*}{ Cas No. } & \multirow{2}{*}{ Model $^{a)}$} & \multicolumn{3}{|c|}{$\log \mathrm{RBA}$} \\
\hline & & & Expt. & Calc. & Deviation \\
\hline Triphenylmethane & $519-73-3$ & 3ERD & -2.25 & -1.63 & 0.62 \\
\hline 3,3',5-Triiodothyropropionic acid & $51-26-3$ & 3ERD & -2.57 & -3.33 & 0.76 \\
\hline 2-Carbethoxy-5,7-dihydroxy-4'-methoxyisoflavone & $15485-76-4$ & 3ERD & -1.37 & -0.73 & 0.64 \\
\hline Kaempferol & $520-18-3$ & 3ERD & -1.54 & -1.22 & 0.32 \\
\hline Naringenin & $480-41-1$ & 3ERD & -2.27 & -1.06 & 1.21 \\
\hline Morin & $6472-38-4$ & 3ERD & -2.96 & -2.99 & 0.03 \\
\hline Apigenin & $520-36-5$ & 3ERD & -1.75 & -1.45 & 0.30 \\
\hline 5,6,7,8-Tetrahydro-2-naphthol & $1125-78-6$ & 3ERD & -3.06 & -2.60 & 0.46 \\
\hline 6-Benzoyl-2-naphthol & $52222-87-4$ & 3ERD & -1.74 & -1.18 & 0.56 \\
\hline L-Epicatechin & $490-46-0$ & 3ERD & -2.42 & -1.01 & 1.41 \\
\hline 8-Hydroxyjulolidine & $41175-50-2$ & 3ERD & N.B. & -3.00 & 1.00 \\
\hline Hematoxylin & $517-28-2$ & 3ERD & -1.52 & -0.55 & 0.97 \\
\hline 3-Hydroxyflavone & $577-85-5$ & 3ERD & N.B. & -4.00 & 0.00 \\
\hline Alternariol & $641-38-3$ & 3ERD & -2.00 & -2.06 & 0.06 \\
\hline Galangin & $548-83-4$ & 3ERD & N.B. & -2.47 & 1.53 \\
\hline 6-Hydroxyflavone & $6665-83-4$ & 3ERD & -2.32 & -1.95 & 0.37 \\
\hline 6-Methoxyflavone & 26964-24-9 & 3ERD & N.B. & -3.14 & 0.86 \\
\hline $3^{\prime}, 4^{\prime}, 7,8$-Tetrahydroxyflavone & $3440-24-2$ & 3ERD & -1.85 & -0.64 & 1.21 \\
\hline 5,6-Dihydroxy-7-methoxyflavone & $29550-13-8$ & 3ERD & -2.77 & -3.42 & 0.65 \\
\hline Formononetin & $485-72-3$ & 3ERD & -1.58 & -0.90 & 0.68 \\
\hline Zearalenone & $17924-92-4$ & 3ERD & 0.45 & 0.76 & 0.31 \\
\hline$\beta$-Zearalanol & $42422-68-4$ & 3ERD & 1.25 & 1.07 & 0.18 \\
\hline$\alpha$-Zearalenol & $36455-72-8$ & 3ERD & 1.72 & 1.21 & 0.51 \\
\hline Zearalanone & $5975-78-0$ & 3ERD & 0.51 & 0.78 & 0.27 \\
\hline Genistein (GEN) & $446-72-0$ & 3ERD & -0.92 & -0.16 & 0.76 \\
\hline 3-Phenylumbelliferone & $6468-96-8$ & 3ERD & -1.64 & -2.02 & 0.38 \\
\hline Iprodione & $36734-19-7$ & 3ERD & N.B. & -4.00 & 0.00 \\
\hline Fenarimol & $60168-88-9$ & 3ERD & -2.75 & -2.55 & 0.20 \\
\hline Carbendazim & $10605-21-7$ & 3ERD & N.B. & -4.00 & 0.00 \\
\hline Triadimefon & $43121-43-3$ & 3ERD & N.B. & -4.00 & 0.00 \\
\hline Mefenacet & $73250-68-7$ & 3ERD & N.B. & -2.82 & 1.18 \\
\hline Imazalil & $35554-44-0$ & 3ERD & N.B. & -4.00 & 0.00 \\
\hline 3-Amino-1-(2,4,6-trichlorophenyl)-5-pyrazolone & $27241-31-2$ & 3ERD & -2.76 & -4.00 & 1.24 \\
\hline 4-Cyclopentylphenol & $1518-83-8$ & 3ERT & -2.60 & -2.72 & 0.12 \\
\hline 2-tert-Butylphenol & $88-18-6$ & 3ERT & N.B. & -4.00 & 0.00 \\
\hline 3,5-Bis(1,1-dimethylethyl)phenol & $1138-52-9$ & 3ERT & N.B. & -2.92 & 1.08 \\
\hline 4-tert-Butylcatechol & $98-29-3$ & 3ERT & -1.72 & -2.05 & 0.33 \\
\hline$p$-Dichlorobenzene & $106-46-7$ & 3ERT & N.B. & -4.00 & 0.00 \\
\hline Pentachlorophenol & $87-86-5$ & 3ERT & N.B. & -4.00 & 0.00 \\
\hline Triallyl trimellitate & $2694-54-4$ & 3ERT & N.B. & -4.00 & 0.00 \\
\hline Diphenyl isophthalate & $744-45-6$ & 3ERT & N.B. & -2.80 & 1.20 \\
\hline Diallyl terephthalate & $1026-92-2$ & 3ERT & N.B. & -4.00 & 0.00 \\
\hline$n$-Butyl-p-aminobenzoate & $94-25-7$ & 3ERT & -3.30 & -4.00 & 0.70 \\
\hline Biphenyl & $92-52-4$ & 3ERT & N.B. & -4.00 & 0.00 \\
\hline 4-(Phenylmethyl)phenol & $101-53-1$ & 3ERT & -1.65 & -2.51 & 0.86 \\
\hline 2,2-Bis-(4-hydroxyphenyl)butane & $77-40-7$ & 3ERT & -0.23 & -1.84 & 1.61 \\
\hline $\begin{array}{l}\text { 4,4'-(Octahydro-4,7-methano-5H-inden-5-ylidene) } \\
\text { bisphenol }\end{array}$ & $1943-97-1$ & 3ERT & 0.34 & 0.36 & 0.02 \\
\hline Rosolic acid & $603-45-2$ & 3ERT & -1.50 & -0.67 & 0.83 \\
\hline 4,4'-(1,4-Phenylenediisopropylidene)bisphenol & $2167-51-3$ & 3ERT & -0.93 & -2.20 & 1.27 \\
\hline 4,4'-Dichlorobenzhydrol & $90-97-1$ & 3ERT & N.B. & -4.00 & 0.00 \\
\hline Benzyl 2,4-dihydroxyphenyl ketone & $3669-41-8$ & 3ERT & -2.67 & -2.61 & 0.06 \\
\hline Tamoxifen & $10540-29-1$ & 3ERT & 1.67 & 1.40 & 0.27 \\
\hline 4-Hydroxybenzophenone & $1137-42-4$ & 3ERT & -1.97 & -1.90 & 0.07 \\
\hline Tris(o-cresyl)phosphate & $78-30-8$ & 3ERT & -1.95 & -1.04 & 0.91 \\
\hline Pararosaniline & $25620-78-4$ & 3ERT & -1.50 & -0.94 & 0.56 \\
\hline Brompheniramine maleate & $980-71-2$ & 3ERT & N.B. & -3.21 & 0.79 \\
\hline Tetraphenylethylene & $632-51-9$ & 3ERT & -0.68 & -0.81 & 0.13 \\
\hline 4-[4-(Trifluoromethyl)phenoxy]phenol & $39634-42-9$ & 3ERT & -2.29 & -2.46 & 0.17 \\
\hline
\end{tabular}


Table 1. Experimental and Calculated $\log$ RBAs for Training and Validation Compounds

\begin{tabular}{|c|c|c|c|c|c|}
\hline \multirow{2}{*}{ Name } & \multirow{2}{*}{ Cas No. } & \multirow{2}{*}{ Model $^{a)}$} & \multicolumn{3}{|c|}{$\log \mathrm{RBA}$} \\
\hline & & & Expt. & Calc. & Deviation \\
\hline Fenofibrate & $49562-28-9$ & 3ERT & N.B. & -2.79 & 1.21 \\
\hline Benzyl 4-chlorophenyl ketone & $1889-71-0$ & 3ERT & N.B. & -4.00 & 0.00 \\
\hline Tris(4-aminophenyl)methanol & $467-62-9$ & 3ERT & -1.42 & -2.00 & 0.58 \\
\hline 2,4,6-Triphenyl-1-hexene & $13964-53-9$ & 3ERT & -1.33 & -2.01 & 0.68 \\
\hline 6-Hydroxyflavanone & $4250-77-5$ & 3ERT & -1.74 & -2.05 & 0.31 \\
\hline 2-Naphthol & $135-19-3$ & 3ERT & -2.98 & -3.27 & 0.29 \\
\hline 6-Bromo-2-naphthol & $15231-91-1$ & 3ERT & -2.67 & -3.03 & 0.36 \\
\hline 1-Naphthol & $90-15-3$ & 3ERT & N.B. & -4.00 & 0.00 \\
\hline 6-Hydroxy-2-naphthyl disulfide & $6088-51-3$ & 3ERT & -1.18 & -1.19 & 0.01 \\
\hline Nafoxidine & $1847-63-8$ & 3ERT & 1.49 & 0.93 & 0.56 \\
\hline 2,7-Dichloro-9-fluorenone & $6297-11-6$ & 3ERT & N.B. & -4.00 & 0.00 \\
\hline 7-Hydroxy-4-(trifluoromethyl)coumarin & $575-03-1$ & 3ERT & -2.46 & -4.00 & 1.54 \\
\hline 2,3-Diphenyl-1-indenone & $1801-42-9$ & 3ERT & -1.11 & -1.18 & 0.07 \\
\hline$\alpha$-Naphtholphthalein & $596-01-0$ & 3ERT & -1.41 & -1.00 & 0.41 \\
\hline Phenobarbital & $50-06-6$ & 3ERT & N.B. & -4.00 & 0.00 \\
\hline Atropine & $51-55-8$ & 3ERT & N.B. & -3.04 & 0.96 \\
\hline Isatoic anhydride & $118-48-9$ & 3ERT & N.B. & -4.00 & 0.00 \\
\hline Hexazinone & $51235-04-2$ & 3ERT & N.B. & -3.07 & 0.93 \\
\hline Quizalofop-ethyl & $76578-14-8$ & 3ERT & N.B. & -3.04 & 0.96 \\
\hline Hexaconazole & 79983-71-4 & 3ERT & N.B. & -4.00 & 0.00 \\
\hline
\end{tabular}

a) The binding affinity of each compound was calculated by using the protein model based on either 3ERD (agonist-bound conformer) or 3ERT (antagonist-bound conformer). $b$ ) Not bound (the maximum displacement of radiolabeled ligand was below $20 \%$ ). $c$ ) When the deviation from the experimental value was estimated, the logRBA value for non-binder (N.B.) was assumed to be -4.0 . d) Not determined (the maximum displacement of radiolabeled ligand was between 20 and $50 \%$ ). $e$ ) When the deviation from the experimental value was estimated, the logRBA value for the compound of which RBA was not determined (N.D.) was assumed to be -3.5 .

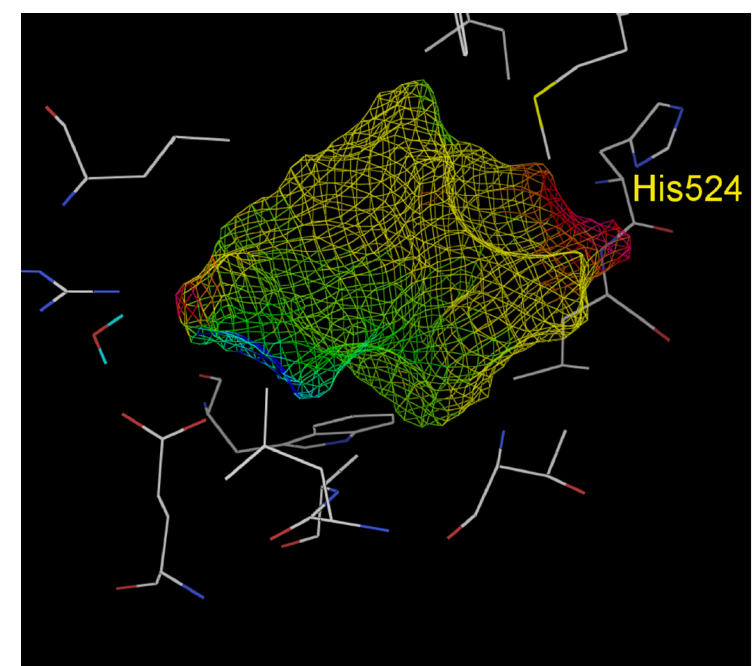

(a)

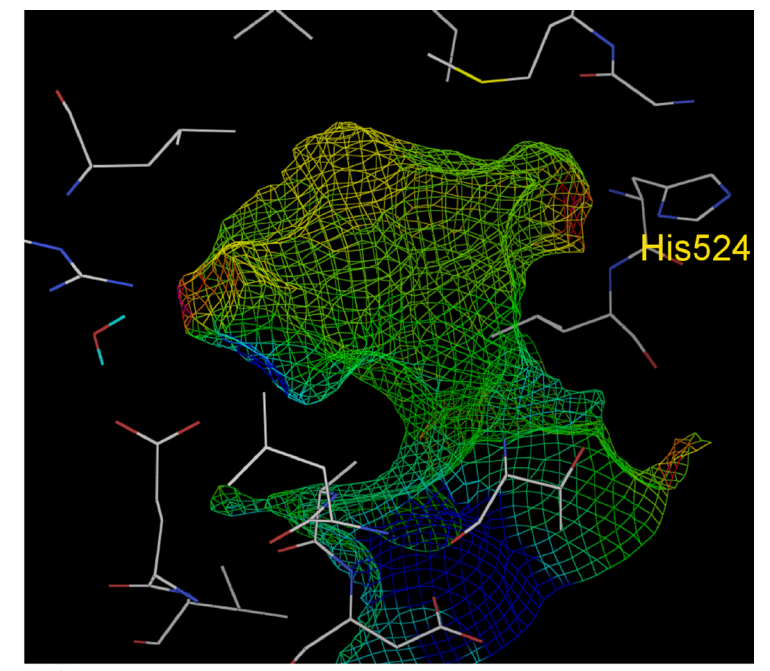

(b)

Fig. 2. Ligand-Binding Cavities of the Two ER $\alpha$ Conformers Used in This Study

(a) Closed cavity of agonist-bound form (3ERD) and (b) open cavity of antagonist-bound form (3ERT). The 3D grid is represented as a bird-cage model. Hydrogens in the protein are omitted for clarity. His524 is the neutral state in this figure.

the ligand-binding region using the CALGRID program (Fig. 2). ${ }^{4,43)}$ Then, the ADAM docking calculation of database compounds to each ER $\alpha$ model was executed with the default parameters. Some compounds in the database had no $\mathrm{H}$-bonding atom. The original ADAM used the H-bonding pattern between ligand and protein as an initial clue, so it could not calculate completely hydrophobic ligands. However, the current version of ADAM can perform the docking of such compounds by matching the aromatic rings or aliphatic groups in the ligand with hydrophobic sites of a target protein (details will be reported elsewhere). The top ten docking modes for each compound were subjected to structure optimization by our energy-minimization program BLUTO, ${ }^{22)}$ to consider the local motion of protein side chains within $7 \AA$ around the ligand-binding site.

Calculation of KeyScore Terms KeyScore $^{17)}$ is an empirical scoring function based on the assumption of a masterequation; that is, it is assumed that different contributions to the stability of a complex can be calculated separately, and summation of all these contributions results in the binding 
score. KeyScore is described as below $\left(c_{1}-c_{8}\right.$ are linear coefficients):

$$
\begin{aligned}
\text { KeyScore }= & c_{1} \Delta N_{\text {ligandrot }}+c_{2} \log P_{\text {ligand }}+c_{3} \log D_{\text {protein }} \\
& +c_{4} \Delta E_{\mathrm{vdW}}+c_{5} \Delta E_{\mathrm{elc}}+c_{6} \Delta H B+c_{7} \Delta E_{\text {confligand }}+c_{8}
\end{aligned}
$$

In the above function, the first five terms are included in the original KeyScore; the details will be reported elsewhere. Their definitions are summarized here very briefly. $\Delta N_{\text {ligandrot }}$ is the conformational entropic change of the ligand between isolated and bound states. This term can be evaluated on the basis of the number of ligand rotatable bonds whose motions are fixed when the ligand partial structure is buried in the protein cavity. $\log P_{\text {ligand }}$ and $\log D_{\text {protein }}$ represent the desolvation energies of the partial surfaces buried due to complex formation for the ligand and protein, respectively. Estimation of these terms is based on $\log P$ of the ligand and $\log D$ of the protein. In this study, the $\log D_{\text {protein }}$ term was excluded (i.e., $c_{3}=0.0$ ), because the accuracy of this term might be limited owing to protein induced-fit motion, and in fact, the preliminary results were not successful (data not shown). $E_{\mathrm{vdW}}$ and $E_{\text {elc }}$ are the intermolecular vdW and electrostatic energies between the protein and ligand, respectively; they are calculated by the AMBER all-atom force field, ${ }^{44)}$ with some modification.

The $\triangle H B$ term was developed in this study. When protein and ligand are separated in the solvent, their hydrophilic groups on the exposed surfaces form several H-bonds with water molecules (i.e., hydrate). Then, when the protein and ligand make a complex, some hydrophilic groups form intermolecular H-bonds directly or mediated by water molecules, and others make unstable contact with the hydrophobic surface of the other molecule. The $\triangle H B$ value represents the change in the number of $\mathrm{H}$-bonds (including hydration number) of hydrophilic groups in both the protein and ligand, from isolated to bound states. The formation of H-bonds is judged by means of distances between the H-bonding heteroatoms and angles among the heteroatoms and an atom that one of the heteroatoms connects to (as a general rule in this method, distance $<3.8 \AA$ and angle $<80$ degrees). Since each hydrophilic group is assumed to be fully hydrated in the isolated state, the $\triangle H B$ term is always greater than or equal to zero, and a smaller $\triangle H B$ contributes to a more stable bound state. Although the binding affinity for ligands with many H-bonding groups is often overestimated when the simple H-bonding number in the bound state is used in the score, the avoidance of such overestimation to some degree can be expected by considering hydration in the isolated state. Note that the $\triangle H B$ term might correlate in part with the $\log P_{\text {ligand }}$ and $\log D_{\text {protein }}$ terms, although we think the explicit consideration of H-bonding separately from the desolvation energy will be necessary to improve the predictive power.

The $\Delta E_{\text {confligand }}$ term, also added in this study, represents the change in conformational energies from the isolated to the bound state for the ligand. The ligand conformational energy was calculated by means of the AMBER force field and our in-house KMF parameter set, which includes extensive force field parameters necessary for calculating the intramolecular energies of small molecules. To obtain the ligand energy in the isolated state, a conformational search was performed in the step of preparing the ligand 3D structure using Key3D, and the lowest energy value among the 15 generated conformations was stored for each stereoisomer.
Classification of Compounds Based on Target Protein Forms We have assumed that each compound interacts more strongly with either the agonist-bound (3ERD) or antagonistbound (3ERT) form and decided to try to predict the binding affinity of each compound on the basis of the ER $\alpha$ form, where it would bind more stably. To select the protein form to be used for the affinity prediction of each compound, a very simple criterion was set; the protein form where the intermolecular vdW energy for the compound was more favorable was adopted. As is well known, the vdW energy correlates very closely with the contact surface area between the two molecules, so the protein form that may contact the compound more extensively should be selected in most cases. If the difference in vdw energies between the two forms was very marginal, i.e., less than $0.2 \mathrm{kcal} / \mathrm{mol}$, the conformational energy of the compound was used as a secondary criterion, so that the protein form where the compound could bind in a more stable conformation took precedence.

Calibration of KeyScore Coefficients The coefficients of KeyScore terms were determined by multiple linear regression (MLR) analysis for each protein form separately. The training compounds for each MLR analysis were selected keeping in mind the diversity of structures and binding affinities. In our scheme, fewer training compounds are required because of the smaller number of variables (KeyScore coefficients). Only 23 compounds were assigned to the training compounds for the 3ERD form and 17 for the 3ERT form (Table 1). The log RBA value for non-binders was assumed to be -4.0 , considering the detection limit of this assay.

For each ER $\alpha$ form, docking calculations assuming two protonation states of His524 have been performed, as described above. All the docking modes generated for the $\operatorname{ER} \alpha$ form with the His524 neutral state were used in the MLR analysis, but in the case of the protein form with the His524 cationic state, docking modes where no H-bond was formed between the compound and the $\mathrm{N} \delta$ atom of His524 were excluded. The purpose of this exclusion was to avoid overestimating electrostatic energy derived from the larger atomic charges of protonated His524, which did not interact with the compound.

For each training compound, tentative KeyScore values were calculated for the selected docking modes by using initial coefficients (i.e., $c_{1}=c_{4}=c_{5}=1.0, c_{2}=-1.0, c_{6}=c_{7}=0.5$, $c_{3}=c_{8}=0.0$ ), and the best docking mode with the most favorable score was determined. Then, MLR analysis was performed for each $\mathrm{ER} \alpha$ form, based on the best docking mode of each training compound, by means of the StatView software, ${ }^{45)}$ and a new candidate for the KeyScore coefficient set was obtained. Using the new coefficient set, the KeyScore values of docking modes were calculated, and the best docking mode was reselected for each compound. Then, MLR analysis was repeated using the reselected docking modes. Thus, the cycle of selecting the best docking modes and the MLR analysis was repeated to reach convergence of the coefficient values. Finally, the prediction equation of ER $\alpha$ binding affinity was obtained for each of the 3ERD and 3ERT forms.

Validation of KeyScore Prediction Each resulting prediction equation was applied to 154 validation compounds, including 109 and 45 compound set classified into the 3ERD and 3ERT groups, respectively (Table 1). Predicted $\log$ RBA values of less than -4.0 were regarded as -4.0 , which was the $\log$ RBA value assumed for non-binders in the experimen- 


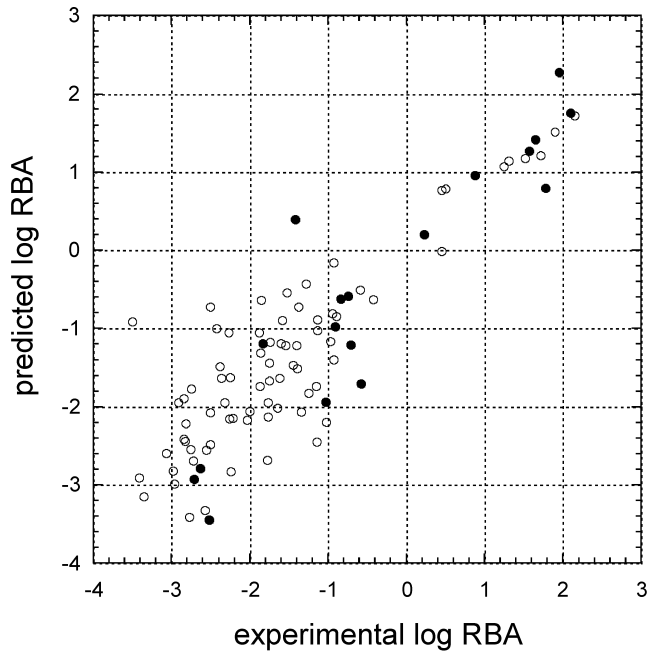

(a)

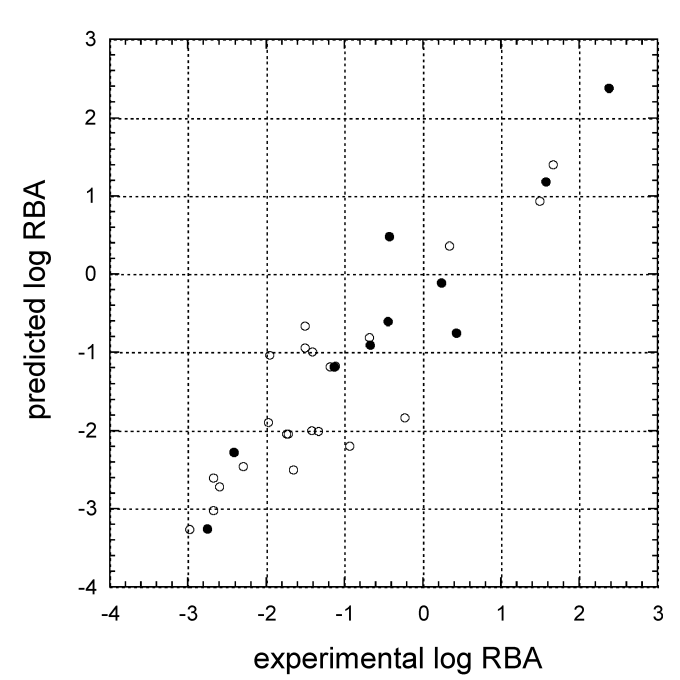

(b)

Fig. 3. Comparison of Experimental and Predicted $\log$ RBA Values

Filled circles: training compounds; open circles: validation compounds. Binding affinity for each compound was predicted by using either the (a) 3ERD or (b) 3ERT model.

tal data.

\section{Results and Discussion}

Resulting Prediction Equations The calibration of KeyScore coefficients for the 3ERD and 3ERT forms provided the following prediction equations.

$$
\begin{aligned}
& \text { 3ERD: } \begin{aligned}
& \log \mathrm{RBA}(\text { calc }) \\
&= 0.086 \Delta N_{\text {ligandrot }}-1.633 \log P_{\text {ligand }} \\
&+0.775 \Delta E_{\mathrm{vdW}}+3.690 \Delta E_{\text {elc }}+0.372 \Delta H B+9.487 \\
& \text { 3ERT: } \log \mathrm{RBA}(\text { calc }) \\
&= 0.457 \Delta N_{\text {ligandrot }}-0.000 \log P_{\text {ligand }} \\
&+ 0.673 \Delta E_{\mathrm{vdW}}+1.977 \Delta E_{\text {elc }}+0.364 \Delta H B+8.200
\end{aligned}
\end{aligned}
$$

The 3ERD prediction model yielded an $r^{2}$ of 0.88 , and the 3ERT model yielded an $r^{2}$ of 0.90 .

The KeyScore coefficients of the 3ERD and 3ERT models displayed different tendencies. For example, the weight of $\Delta N_{\text {ligandrot }}$ in the 3ERD model was considerably smaller than that in 3ERT. This should be because most of the ligands binding to the 3ERD model were small and comparatively rigid, so the conformational entropic term contributed only slightly to the predicted affinity. Also, significant differences of the $\log P_{\text {ligand }}$ and $\Delta E_{\text {elc }}$ weights appeared between the two protein models. It would be complicated to consider these differences qualitatively, since these terms and $\triangle H B$ are mutually interrelated in part. The coefficients of the ligand conformational energies were zero for both protein models. This should not mean that the ligand conformational energy is irrelevant to the binding affinity to $\mathrm{ER} \alpha$, but it would occur because the intramolecular energy difference between the unbound and bound states could not be estimated accurately for ligands with a variety of sizes and structures. As Jorgensen pointed out, ${ }^{46)}$ accurate evaluation of free-energy changes associated with ligand conformation is still difficult for the current force field.

Affinity Prediction of Validation Compounds As in the
Table 2. Comparison of Prediction Results of KeyScore and Other Methods

\begin{tabular}{lcccc}
\hline \hline & \multicolumn{2}{c}{ Training set } & \multicolumn{2}{c}{ Validation set } \\
\cline { 2 - 5 } & $N$ & $r^{2}$ & $N$ & Predictive $r^{2}$ \\
\hline CoMFA $^{a)}$ & 130 & 0.91 & $25^{e)}$ & 0.62 \\
& & & $25^{f}$ & 0.71 \\
6D-QSAR $^{b)}$ & 88 & $0.90^{d)}$ & 18 & 0.89 \\
Raptor & & & 23 & 0.91 \\
KeyScore & 93 & & & \\
3ERD & 23 & 0.88 & 109 & 0.83 \\
3ERT & 17 & 0.9 & 45 & 0.83 \\
\hline
\end{tabular}

a) Ref. 29. b) Ref. 15. c) Ref. 14. d) Cross-validated $r^{2}$. e) Kuiper's dataset. $f$ ) Waller's dataset.

case of the training compounds, each validation compound was classified into either the 3ERD or 3ERT group, and its predictive $\log \mathrm{RBA}$ was calculated on the basis of the corresponding prediction equation (Table 1). For the 109 validation compounds in the 3ERD group, a predictive $r^{2}$ of 0.83 was obtained, and for the 45 validation compounds in the 3ERT group, a predictive $r^{2}$ of 0.83 was obtained (Fig. 3). Compared to the original universal KeyScore, which yielded a predictive $r^{2}$ of 0.44 when using the 3ERD model, the ER $\alpha$-targeted score in this study demonstrated significantly improved predictive power.

For all of the 154 validation compounds except testosterone, the predicted $\log$ RBAs deviated by less than 2.0 from the experimental values (Table 1). The cases with larger differences between calculation and experiment, including testosterone, will be discussed later. Table 2 compares the prediction results of our scheme and those of other methods. The predictive power of our scheme is apparently superior to that of CoMFA (predictive $\left.r^{2}=0.62-0.71, N=25\right)$, ${ }^{29)}$ while it seems slightly lower than those of 6D-QSAR (predictive $r^{2}=0.89$, $N=18)^{15)}$ and Raptor (predictive $r^{2}=0.91, N=23$ ). ${ }^{14)}$ QSAR approaches, including the above, generally use a large number of 

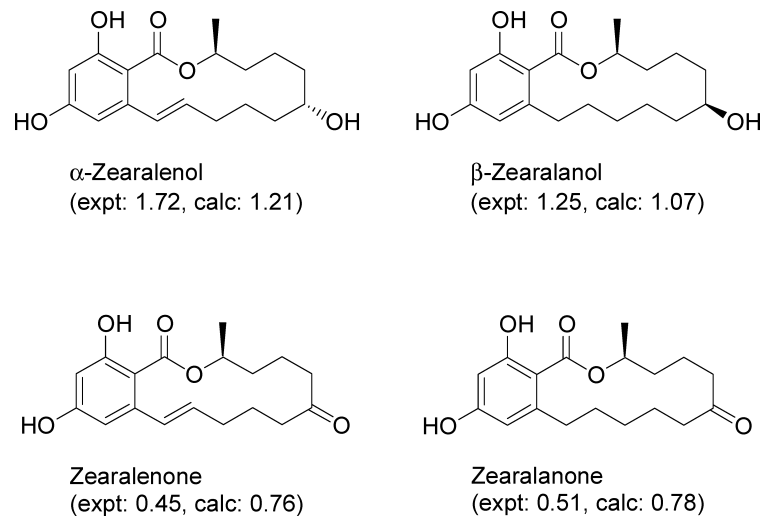

Fig. 4. Chemical Structures and $\log$ RBA Values of Zearalenol and Analogs

explanatory variables, because every weighting value to each property at each grid point generated in the $3 \mathrm{D}$ space surrounding the receptor cavity model is handled as a variable. Therefore, the calculated binding affinities can be fit easily to the experimental ones for these methods, although many training compounds are needed to calibrate so many variables (e.g., 88 training compounds were used for 6D-QSAR and 93 for Raptor). Our approach could construct a predictive scheme with satisfactory performance using far fewer training compounds than the other methods (23 training compounds for the 3ERD model and 17 for 3ERT). Considering that only a few active compounds are known for many of the intriguing drug targets, our approach, which requires fewer known ligands, would be more practical and useful. Furthermore, while in principle the high predictive power of QSAR approaches would be displayed only for the structure classes included in the training set, our energy-based method is not directly affected by the structural frameworks of training compounds; so acceptable predictive power can also be expected for test structures beyond the training structure classes. In reality, for zearalenol and its analogs, the structure class of which was not included in the training set, our prediction scheme demonstrated good predictive power; the maximal deviation from the experimental $\log$ RBA was only 0.51 (Fig. 4).

For several compounds, the calculated log RBA deviated significantly $(>1.50)$ from the experiment (Fig. 5). Among them, resveratrol and 3',4',7-trihydroxyisoflavone each have three hydroxyl groups. In our experience, it is often difficult to estimate adequately the electrostatic interaction and the compensation by desolvation cost for ligands with several hydroxyl groups, and this problem should cause overestimation of binding affinities in the present case. As for 2,2-bis-(4hydroxyphenyl)butane, the correct docking mode might not be ranked first owing to the failure to fully consider local protein movement, and for 7-hydroxy-4-(trifluoromethyl)coumarin, the weak affinity of the small compound could not be estimated properly. Most of the non-binders could be predicted adequately, but several false positives were included (predicted $\log$ RBA of non-binder $>-2.50$ ) (Fig. 5). For galangin, the overestimation of energy relevant to three hydroxyl groups is a possible cause of the false positive, as in the case of $3^{\prime}, 4^{\prime}, 7$-trihydroxyisoflavone. As for $6 \beta$-hydroxytestosterone and testosterone, their docking modes were sufficiently reasonable and consistent with the cocrystal mode of E2. The structures
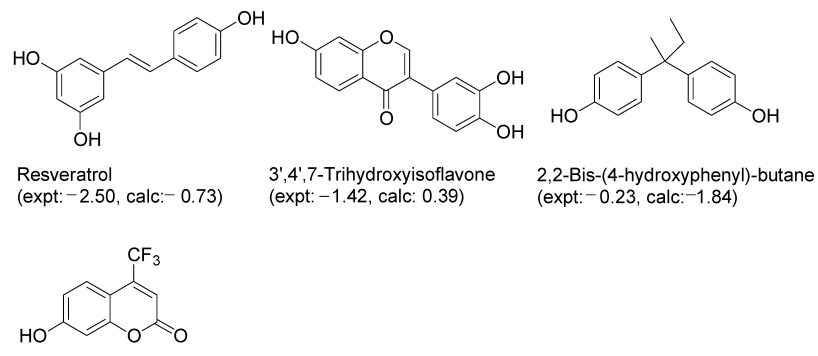

7-Hydroxy-4-(trifluoromethyl)coumarin (expt: -2.46 , calc: -4.00$)$

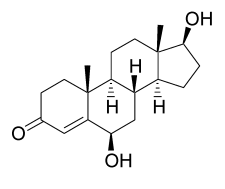

$6 \beta$-Hydroxytestosterone (expt: N.B., calc:- 2.26 )
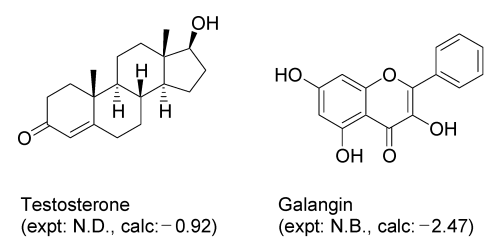

Galangin

(expt: N.B., calc: -2.47 )
Fig. 5. Chemical Structures and $\log$ RBA Values of Outliers and False Positives

of these compounds fitted very closely with the protein cavity in appearance, so the vdW and electrostatic energies were very favorable. This should cause overestimation of the binding affinities. We have to keep trying to properly estimate the difference in binding affinities between estradiol analogs and testosterone analogs.

Predicted Docking Modes One of the merits of our method is that the correct docking modes can be generated in most cases. Figure 6 compares the ER $\alpha$ complex structures between the cocrystal and docking modes. For the ligands included in the cocrystal structures, root-mean-square deviations (R.M.S.D.) for all non-hydrogen atoms in the docking model from the cocrystal coordinates were $0.32 \AA, 0.42 \AA$, $1.17 \AA$ and $0.62 \AA$ for DES, E2, OHT and genistein (GEN; Fig. 6a), respectively. Generally, the docking mode with R.M.S.D. $<2.0 \AA$ is judged as correct, so it can be said that our docking models predicted by ADAM were successful. In the docking model of GEN (Fig. 6a), the binding position, conformation and four $\mathrm{H}$-bonds were closely consistent with those in the cocrystal structure. As for the docking model of $\alpha$-zearalenol (Fig. 6b), of which cocrystal structure has not been elucidated, two hydroxyl groups were positioned in the vicinity of those in the cocrystal mode of E2 and each group formed an H-bond with Glu353 and His524, respectively. The $\alpha$-zearalenol in the docking model occupied the cavity of ER agonist-bound form wholly, just like the E2 cocrystal mode. In the docking model of nafoxidine (Fig. 6c), the methoxy group bound to the site near the binding site of the hydroxyl group of OHT, and formed two weak H-bonds with Arg394 and water molecule. Tertiary amine nitrogen, ether oxygen and three aromatic rings in the nafoxidine docking model were positioned close to those in the OHT cocrystal structure. Thus, the comparison between the cocrystal and docking modes suggests the validity of the ADAM docking calculation, and this ability to generate the correct docking modes should greatly contribute to the high predictive power of our approach to estimate the binding affinities. Meanwhile, in many QSAR studies, the binding coordinates in cocrystal structures have been used for ligand alignment, and such approaches should have only poor applicability and reliability for ligand classes 


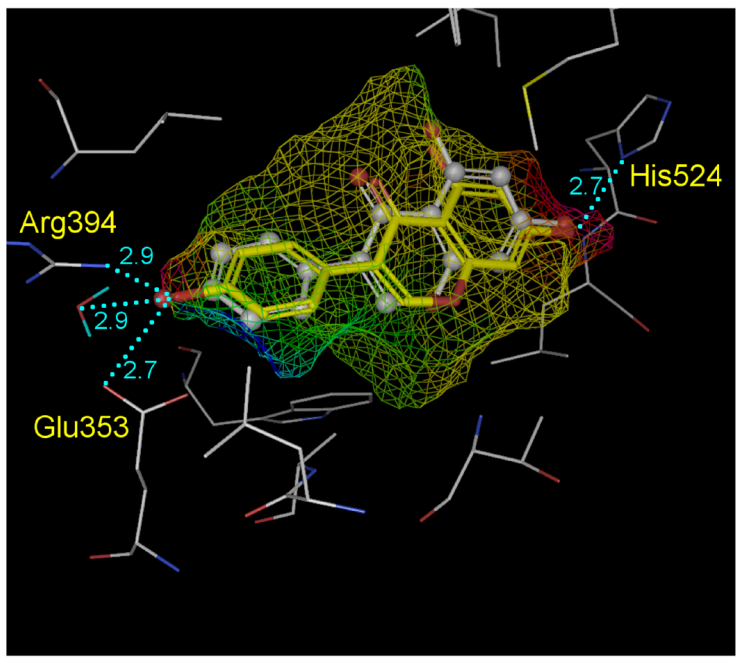

(a)

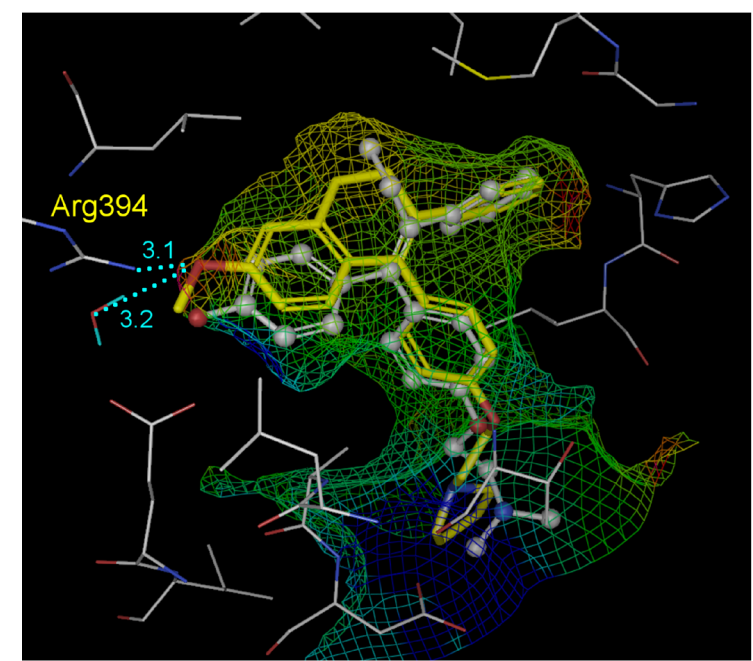

(c)

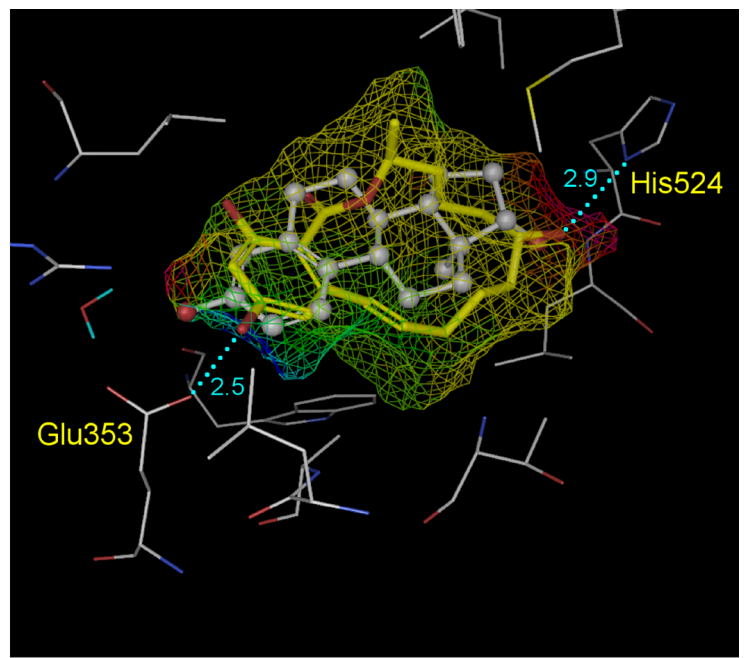

(b)

Fig. 6. Docking Modes of GEN, $\alpha$-Zearalenol, and Nafoxidine, Compared with Cocrystal Structures of Known Inhibitors

(a) Docking and cocrystal modes of GEN (PDB: 1X7R) ${ }^{47}$ ) in the 3ERD form (R.M.S.D. $0.62 \AA$ ), (b) docking mode of $\alpha$-zearalenol and cocrystal structure of E2 (PDB: 1ERE) ${ }^{37)}$ in the 3ERD form, (c) docking mode of nafoxidine and cocrystal structure of OHT in the 3ERT form. The docking modes are shown as cylinder models, and cocrystal structures are as ball-and-stick ones. The shape of the ligand-binding region is displayed as a bird-cage model. Dotted lines represent intermolecular H-bonds formed in docking modes, and the H-bonding distances ( $\AA$ unit) are also noted. Hydrogens in the protein and ligands are omitted for clarity.

of which the cocrystal structures are unavailable. Some QSAR approaches use the docking results. For example, 6D-QSAR used the results of the flexible docking by Yeti, ${ }^{48)}$ but each docking simulation required high computational costs. ${ }^{15)}$ The CPU times of our method were $0.5-60 \mathrm{~s}$ for the ADAM docking of each compound and $6-17 \mathrm{~s}$ for the Bluto optimization of each docking mode on our Linux machine (Intel Xeon CPU, 3.06 GHz).

Effects of the MCMP Approach In this study, we have used as the docking targets two cocrystal structures in which the $\mathrm{ER} \alpha$ conformations were very different, and the binding affinity of each chemical was estimated on the basis of either of the two protein conformers. The classification of the compounds into two groups (the 3ERD and 3ERT groups), based mainly on the vdw energies, is shown in Table 1 . Most of the agonists such as steroids and phytohormones were classified in the 3ERD (agonist-bound form) group, and antagonists with larger sizes were classified in the 3ERT (antagonist-bound form) group, adequately. However, it should be noted that this classification is intended to yield appropriate affinity prediction for each compound by adopting the protein model to which the compound might contact more extensively and stably, but not to try to discriminate between agonists and antagonists. To examine the effects of this multiple-conformations (MC) approach, we have attempted to make predictions based on only the 3ERT form using all 40 training compounds. Compared with the MC approach, the single-conformation (SC) approach based on only the 3ERT model had considerably lower predictive power; the predictive $r^{2}$ for the 154 validation compounds was 0.64 (Table 3 ). We consider that the main reason for this is as follows: when a small agonist was docked in the antagonist-bound ER $\alpha$ form (3ERT), the ligand partial structure, which should actually be in contact with the bottom of the closed cavity of the agonist-bound form, could 
Table 3. Comparison of Prediction Results of KeyScore, Based on Three Schemes

\begin{tabular}{lc}
\hline \hline & $r^{2}$ for training set $(N)$ \\
\hline Multiple-conformations-multiple-protonation (MCMP) approach & \\
3ERD & $0.88(23)$ \\
3ERT & $0.90(17)$ \\
Single-conformation-multiple-protonation (SCMP) approach $^{a)}$ & $0.80(40)$ \\
3ERT & $0.83(109)$ \\
Multiple-conformations-single-protonation (MCSP) approach & \\
3ERD & $0.83(45)$ \\
3ERT & $0.90(17)$ \\
\hline
\end{tabular}

a) Only one ER $\alpha$ model was used in the prediction calculation. $b$ ) The imidazole of His524 was assumed to be neutral (only the $N \varepsilon$ atom was protonated).

not interact with the open cavity of 3ERT, so the interaction energy of such a ligand was underestimated. As for the SC approach based on 3ERD, stable docking modes of large antagonists were not generated.

In addition to considering two protein conformations, the two protonation states of His524 at the site where the steroid D-ring binds were also considered for each protein conformer. Table 3 compares the predictive powers of this multipleprotonation (MP) approach and the single-protonation (SP) approach, in which His524 was assumed to be always in the neutral state (i.e., only the $N \varepsilon$ atom was protonated). In the 3ERD form, it was shown that considering the two protonation states contributed to improving the predictive power (predictive $r^{2}$ : 0.76 for the SP approach, 0.83 for the MP approach). On the other hand, the MP approach had no effect on the 3ERT model (predictive $r^{2}: 0.83$ for the SP and MP approaches). The reason is that few compounds in the training and validation sets for the 3ERT model formed $\mathrm{H}$-bonds with His524.

In recent years, a variety of docking methods taking into account the conformational flexibility of target protein have been reported; ${ }^{39,49)}$ for example, the docking of the ligand to ensembles of multiple protein structure ${ }^{50-52)}$ or an average protein grid, ${ }^{53,54)}$ methods using soft docking potentials, ${ }^{55)}$ approaches exploring side-chain rotamer libraries, ${ }^{56,57)}$ and current unique methods such as IFREDA ${ }^{58)}$ and the iterative coupling of Glide and Prime. ${ }^{59)}$ Every method has both merits and demerits, and there is no decisive method that also shows outstanding effects on virtual screening. In our MCMP approach, the major protein movement and the change in protonation states accompanied by ligand binding are taken into account as discrete protein models, and the local induced-fit motion in each protein model is handled during the ADAM docking calculation and the succeeding structure optimization step. Our approach should be classified as ensemble docking. It has been pointed out that, in ensemble docking, generally, the use of more protein conformers often increases not only the computational costs but also the ambiguity of the docking results (i.e., several docking modes from the different protein conformers get equally good scores) and false positives. ${ }^{39,50,60)}$ One of the advantages of our approach is that it needs only relatively few protein models that have very different conformations from one another, because the local movement of proteins is considered during docking and optimization. Furthermore, for the problem of selection from the docking modes based on several protein models, this study demonstrated a successful example using very simple criteria on the basis of vdW interaction.

Nevertheless, the MCMP approach also has several problems, most of which are shared by the other ensemble docking methods. First, for ligands that would bind to very different protein conformers from the models used in docking, it is anticipated that the predicted docking modes and affinities should be less reliable. Additional use of cocrystal structures with different conformations or, if such various crystal structures are not available, appropriate conformational sampling of the target protein is needed to address the problem. A variety of computational methods for conformational sampling, including molecular dynamics simulation and normal mode analysis, have been studied intensively, although difficult problems still remain regarding generation and selection of necessary and sufficient protein conformations. A second problem is the increase in computational cost in proportion to the number of protein models. To partially solve this problem, we have been developing a method for dealing with several protonation states simultaneously in a protein model.

\section{Conclusion}

We have constructed an effective scheme that predicts the ligand binding affinities to ER $\alpha$ by means of the docking method ADAM and an improved version of KeyScore. This predictive scheme uses the MCMP approach, which considers the combination of several protein conformations and several protonation states. The binding affinities for a number of validation compounds were predicted with a high degree of accuracy by our approach (predictive $r^{2}=0.83, N=154$ in total). Since our energy-based method includes far fewer variables to be calibrated compared with general QSAR approaches, it requires fewer training compounds, and it is expected to work effectively even for target proteins with fewer known ligands. Furthermore, even if our approach is applied to the test compounds with different frameworks from the training compounds, a comparable predictive power is expected because the structural features of the training compounds are not reflected directly in the resulting prediction equation. Also, the docking modes generated by ADAM provide useful suggestions for planning the structure modification to improve its activities and properties.

The goal of KeyScore is to construct a universal prediction formula that shows satisfactory predictive power in extensive protein targets. However, the accuracy of each score term is apparently insufficient at present due to the basic problems shared among all docking and scoring methods (see Introduction); therefore, we consider that the calibration 
of score weights for every protein target may be practically meaningful. An efficient prediction scheme was generated in the $\operatorname{ER} \alpha$ case, so we are now trying to apply our approach to other protein systems. While addressing the basic problems for generating a universal prediction score, we aim also at accumulating the know-how for every characteristic protein system and at proposing practical and useful solutions for each case.

Acknowledgement We thank Dr. Yumi Akahori at the Chemicals Evaluation and Research Institute for assistance and advise in handling the binding data.

\section{References and Notes}

1) Klebe G., Drug Discov. Today, 11, 580-594 (2006).

2) Scapin G., Curr. Pharm. Des., 12, 2087-2097 (2006).

3) Ekins S., Mestres J., Testa B., Br. J. Pharmacol., 152, 9-20 (2007).

4) Moitessier N., Englebienne P., Lee D., Lawandi J., Corbeil C. R., Br. J. Pharmacol., 153(Suppl 1), S7-S26 (2008).

5) Sousa S. F., Fernandes P. A., Ramos M. J., Proteins, 65, 15-26 (2006).

6) Kontoyianni M., Madhav P., Suchanek E., Seibel W., Curr. Med. Chem., 15, 107-116 (2008).

7) Leach A. R., Shoichet B. K., Peishoff C. E., J. Med. Chem., 49, 5851-5855 (2006)

8) Kollman P., Chem. Rev., 93, 2395-2417 (1993).

9) Hansson T., Åqvist J., Protein Eng., 8, 1137-1144 (1995).

10) Zhang T., Koshland D. E. Jr., Protein Sci., 5, 348-356 (1996).

11) Novotny J., Bruccoleri R. E., Davis M., Sharp K. A., J. Mol. Biol., 268, 401-411 (1997).

12) Coupez B., Lewis R. A., Curr. Med. Chem., 13, 2995-3003 (2006).

13) Cramer R. D., Patterson D. E., Bunce J. D., J. Am. Chem. Soc., 110, 5959-5967 (1988).

14) Lill M. A., Vedani A., Dobler M., J. Med. Chem., 47, 6174-6186 (2004).

15) Vedani A., Dobler M., Lill M. A., J. Med. Chem., 48, 3700-3703 (2005).

16) Takamatsu Y., Itai A., Proteins, 33, 62-73 (1998).

17) Takamatsu Y., Mizutani M. Y., Itai A. (in preparation)

18) Seifert M. H., Drug Discov. Today, 14, 562-569 (2009).

19) Jansen J. M., Martin E. J., Curr. Opin. Chem. Biol., 8, 359-364 (2004).

20) Yamada M., Itai A., Chem. Pharm. Bull., 41, 1200-1202 (1993).

21) Mizutani M. Y., Tomioka N., Itai A., J. Mol. Biol., 243, 310-326 (1994).

22) Mizutani M. Y., Takamatsu Y., Ichinose T., Nakamura K., Itai A., Proteins, 63, 878-891 (2006).

23) Mizutani M. Y., Itai A., J. Med. Chem., 47, 4818-4828 (2004).

24) Iwata Y., Arisawa M., Hamada R., Kita Y., Mizutani M. Y., Tomioka N., Itai A., Miyamoto S., J. Med. Chem., 44, 1718-1728 (2001).

25) Ishioka T., Kubo A., Koiso Y., Nagasawa K., Itai A., Hashimoto Y., Bioorg. Med. Chem., 10, 1555-1566 (2002).

26) Tanaka A., Konno M., Muto S., Kambe N., Morii E., Nakahata T., Itai A., Matsuda H., Blood, 105, 2324-2331 (2005).

27) Nilsson S., Gustafsson J. A., Crit. Rev. Biochem. Mol. Biol., 37, $1-28$ (2002).

28) Kavlock R. J., Daston G. P., DeRosa C., Fenner-Crisp P., Gray L. E., Kaattari S., Lucier G., Luster M., Mac M. J., Maczka C., Miller R., Moore J., Rolland R., Scott G., Sheehan D. M., Sinks T., Tilson H. A., Environ. Health Perspect., 104 (Suppl. 4), 715-740 (1996).

29) Shi L. M., Fang H., Tong W., Wu J., Perkins R., Blair R. M.,
Branham W. S., Dial S. L., Moland C. L., Sheehan D. M., J. Chem. Inf. Comput. Sci., 41, 186-195 (2001).

30) Hong H., Tong W., Fang H., Shi L., Xie Q., Wu J., Perkins R., Walker J. D., Branham W., Sheehan D. M., Environ. Health Perspect., 110, 29-36 (2002).

31) Tong W., Xie Q., Hong H., Shi L., Fang H., Perkins R., Environ. Health Perspect., 112, 1249-1254 (2004).

32) Yang J. M., Shen T. W., Proteins, 59, 205-220 (2005).

33) Demyttenaere-Kovatcheva A., Cronin M. T., Benfenati E., Roncaglioni A., Lopiparo E., J. Med. Chem., 48, 7628-7636 (2005).

34) Zhu B. T., Han G. Z., Shim J. Y., Wen Y., Jiang X. R., Endocrinology, 147, 4132-4150 (2006).

35) Wolohan P., Reichert D. E., Steroids, 72, 247-260 (2007).

36) Knox A. J., Meegan M. J., Sobolev V., Frost D., Zisterer D. M., Williams D. C., Lloyd D. G., J. Med. Chem., 50, 5301-5310 (2007).

37) Brzozowski A. M., Pike A. C., Dauter Z., Hubbard R. E., Bonn T., Engström O., Ohman L., Greene G. L., Gustafsson J. A., Carlquist M., Nature (London), 389, 753-758 (1997).

38) Shiau A. K., Barstad D., Loria P. M., Cheng L., Kushner P. J., Agard D. A., Greene G. L., Cell, 95, 927-937 (1998).

39) B-Rao C., Subramanian J., Sharma S. D., Drug Discov. Today, 14, 394-400 (2009)

40) Akahori Y., Nakai M., Yamasaki K., Takatsuki M., Shimohigashi Y., Ohtaki M., Toxicol. In Vitro, 22, 225-231 (2008). Data from http://www.safe.nite.go.jp/japan/db.html (Japanese)

41) Mizutani M. Y., Nakamura K., Ichinose T., Itai A., Chem. Pharm. Bull., 54, 1680-1685 (2006).

42) Tomioka N., Itai A., Iitaka Y., J. Comput. Aided Mol. Des., 1, 197-210 (1987).

43) Tomioka N., Itai A., J. Comput. Aided Mol. Des., 8, 347-366 (1994).

44) Cornell W. D., Cieplak P., Bayly C. I., Gould I. R., Merz K. M. Jr., Ferguson D. M., Spellmeyer D. C., Fox T., Caldwell J. W., Kollman P. A., J. Am. Chem. Soc., 117, 5179-5197 (1995).

45) StatView version 5.0, SAS Institute, Inc.

46) Tirado-Rives J., Jorgensen W. L., J. Med. Chem., 49, 5880-5884 (2006).

47) Manas E. S., Xu Z. B., Unwalla R. J., Somers W. S., Structure, 12, 2197-2207 (2004).

48) Vedani A., Huhta D. W., J. Am. Chem. Soc., 112, 4759-4767 (1990).

49) Cozzini P., Kellogg G. E., Spyrakis F., Abraham D. J., Costantino G., Emerson A., Fanelli F., Gohlke H., Kuhn L. A., Morris G. M., Orozco M., Pertinhez T. A., Rizzi M., Sotriffer C. A., J. Med. Chem., 51, 6237-6255 (2008).

50) Totrov M., Abagyan R., Curr. Opin. Struct. Biol., 18, 178-184 (2008).

51) Sutherland J. J., Nandigam R. K., Erickson J. A., Vieth M., J. Chem. Inf. Model., 47, 2293-2302 (2007).

52) Carlson H. A., Curr. Opin. Chem. Biol., 6, 447-452 (2002).

53) Knegtel R. M., Kuntz I. D., Oshiro C. M., J. Mol. Biol., 266, 424440 (1997).

54) Bottegoni G., Kufareva I., Totrov M., Abagyan R., J. Med. Chem., 52, 397-406 (2009).

55) Jiang F., Kim S. H., J. Mol. Biol., 219, 79-102 (1991).

56) Leach A. R., J. Mol. Biol., 235, 345-356 (1994).

57) Alberts I. L., Todorov N. P., Dean P. M., J. Med. Chem., 48, 65856596 (2005).

58) Cavasotto C. N., Abagyan R. A., J. Mol. Biol., 337, 209-225 (2004).

59) Sherman W., Day T., Jacobson M. P., Friesner R. A., Farid R., J. Med. Chem., 49, 534-553 (2006).

60) Barril X., Morley S. D., J. Med. Chem., 48, 4432-4443 (2005). 